\title{
On the collapsing along deformations of hyperbolic cone 3 -manifolds
}

\author{
Alexandre Paiva Barreto
}

\begin{abstract}
This article focuses on deformations of hyperbolic cone structures under the assumption that the length of the singularity remains uniformly bounded during the deformation. Let $M$ be a closed, orientable, and irreducible 3-manifold, and let $\Sigma$ be an embedded link in $M$. For a collapsing sequence of hyperbolic cone structures with topological type $(M, \Sigma)$ and with uniformly bounded lengths of singularities, we prove that $M$ is either Seifert fibered or a Sol manifold.
\end{abstract}

\section{Introduction}

In this article we study deformations of hyperbolic cone structures on a closed, orientable, and irreducible 3-manifold $M$ which are singular along a fixed embedded link $\Sigma=\Sigma_{1} \sqcup \cdots \sqcup \Sigma_{l}$. Unlike complete hyperbolic structures, which are rigid by the Mostow theorem, the hyperbolic cone structures could be deformed (see [HK2]). The difficulty in understanding these deformations lies in the possibility of degenerating the structure. In other words, the Hausdorff-Gromov limit (see Section 2 for the definition) of the deformation could be only an Alexandrov space which may have dimension strictly smaller than 3 , although its curvature remains bounded from below by -1 (see $[$ Koj]).

The works of Kojima [Koj], Hodgson-Kerckhoff [HK], and Fuji [Fuj] show that the degeneration of the hyperbolic cone structures occurs if and only if the singular link of these structures intersects itself during the deformation. When the cone angles vary between 0 and $\pi$, the Dirichlet polyhedron of the hyperbolic cone structures are convex and this fact can be used to avoid self-intersections of the singular link during deformations (see $[\mathrm{Koj}]$ ). In this article, this restrictive assumption will not be made and the cone angles will be allowed to vary until $2 \pi$.

We are interested in studying the following question which was proposed by W. Thurston in the 1980s.

QUESTION 1

Given a closed and orientable hyperbolic 3-manifold $M$, let $\Sigma$ be a simple closed geodesic $\Sigma$ in $M$. Can the hyperbolic structure of $M$ be deformed to the complete

Kyoto Journal of Mathematics, Vol. 56, No. 3 (2016), 539-557

DOI 10.1215/21562261-3600166, (C) 2016 by Kyoto University

Received October 10, 2014. Revised July 16, 2015. Accepted July 30, 2015.

2010 Mathematics Subject Classification: 57M50.

Author's work partially supported by Projeto Regular FAPESP No. 2014/23398-2. 
hyperbolic structure on $M-\Sigma$ through a path $M_{\alpha}$ of hyperbolic cone structures with topological type $(M, \Sigma)$ and parameterized by the cone angles $\alpha \in[0,2 \pi]$ ?

If the deformation proposed by Thurston in Question 1 exists, then it is a consequence of Thurston's hyperbolic Dehn surgery theorem that the length of the singular link must converge to zero. In particular, it follows that the length of the singular link remains uniformly bounded during the deformation. This conclusion gives us a necessary condition for the existence of Thurston's deformation. For this reason, we shall focus on deformations of hyperbolic cone structures with this additional hypothesis on the singularity length. We note that this assumption is automatically verified when the holonomy representations of the hyperbolic cone structures are convergent and we remark that it avoids the undesirable case where the singular link becomes dense in the limit Alexandrov space.

When a convergent (in the Hausdorff-Gromov sense) sequence of hyperbolic cone manifolds does not collapse, the limit Alexandrov space must have dimension 3. In this case, several geometric techniques are known to study the topological type of $M$. In the collapsing case, however, most of the geometric information is lost. This happens because the dimension of the limit Alexandrov space is strictly smaller than 3 (see Lemma 14).

As mentioned, this article deals with collapsing deformations of hyperbolic cone structures. Our aim is to find conditions to avoid the possibility of collapsing. The main theorem of this article is the following one (see Section 3 for the definition of collapse).

\section{THEOREM 2}

Given a closed, orientable, and irreducible 3-manifold $M$, let $\Sigma=\Sigma_{1} \sqcup \cdots \sqcup \Sigma_{l}$ be an embedded link in $M$. Suppose that there is a converging sequence $M_{i}$ of hyperbolic cone manifolds with topological type $(M, \Sigma)$ and having cone angles $\alpha_{i j} \in(0,2 \pi]$. Denote by $\mathcal{L}_{M_{i}}\left(\Sigma_{j}\right)$ the length of the connected component $\Sigma_{j}$ of $\Sigma$ in the hyperbolic cone manifold $M_{i}$. If the sequence $M_{i}$ collapses and

$$
\sup \left\{\mathcal{L}_{M_{i}}\left(\Sigma_{j}\right) ; i \in \mathbb{N} \text { and } j \in\{1, \ldots, l\}\right\}<\infty,
$$

then $M$ is Seifert fibered or a Sol manifold.

\section{REMARK 3}

The hypothesis (1) on the length of the singularity is fundamental to treating the case where the limit space is not compact. For the compact case, it can be replaced by the weaker hypothesis that the singular locus does not become dense in the limit Alexandrov space.

As a consequence of Theorem 2, we shall obtain the following results yielding information on Thurston's question. 


\section{COROLLARY 4}

Given a closed and orientable hyperbolic 3-manifold $M$, let $\Sigma$ be a finite union of disjoint simple closed geodesics in $M$. Consider a (angle-decreasing) deformation $M_{\alpha}$ of this structure along a continuous path of hyperbolic cone structures with topological type $(M, \Sigma)$ and having cone angles $\alpha \in(L, 2 \pi] \subset[0,2 \pi]$ (the same for all components of $\Sigma$ ). If

$$
\sup \left\{\mathcal{L}_{M_{\alpha}}\left(\Sigma_{j}\right) ; \alpha \in(L, 2 \pi] \text { and } j \in\{1, \ldots, l\}\right\}<\infty,
$$

then every convergent sequence $M_{\alpha_{i}}$, with $\alpha_{i}$ converging to $L$, does not collapse.

\section{COROLLARY 5}

Given a closed and orientable hyperbolic 3-manifold $M$, let $\Sigma$ be a finite union of disjoint simple closed geodesics in $M$. Consider a (angle-decreasing) deformation $M_{\alpha}$ of this structure along a continuous path of hyperbolic cone structures with topological type $(M, \Sigma)$ and having cone angles $\alpha \in(L, 2 \pi] \subset[0,2 \pi]$ (the same for all components of $\Sigma)$.

Suppose that there is a sequence $M_{\alpha_{i}}$ converging to a compact Alexandrov space, with $\alpha_{i}$ converging to $L$. If the singular locus does not become dense in the limit space, then the sequence $M_{\alpha_{i}}$ does not collapse.

Note that Corollaries 4 and 5 rule out the possibility of collapsing through the deformation, but a degeneration of the structure can still happen. When $L \leq \pi$, Kojima's [Koj] work says that the deformation can be pushed to 0 .

\section{Metric geometry}

In this section, we recall some definitions about Alexandrov spaces and HausdorffGromov convergence. We refer to [BBI], [BGP], and [Gro] for details.

Given a metric space $Z$, the metric on $Z$ will always be denoted by $d_{Z}(\cdot, \cdot)$. The open (resp., closed) ball of radius $r>0$ about a subset $A$ of $Z$ will be denoted by

$$
\begin{aligned}
& B_{Z}(A, r)=\bigcup_{a \in A}\left\{z \in Z ; d_{Z}(z, a)<r\right\} \\
& \left.\quad \text { resp., } B_{Z}[A, r]=\bigcup_{a \in A}\left\{z \in Z ; d_{Z}(z, a) \leq r\right\}\right) .
\end{aligned}
$$

A metric space $Z$ is called a length space (and its metric is called intrinsic) when the distance between every pair of points in $Z$ is given by the infimum of the lengths of all rectifiable curves connecting them. When a minimizing geodesic between every pair of points exists, we say that $Z$ is complete. For every $k \in \mathbb{R}$, denote by $\mathbb{M}_{k}^{2}$ the complete and simply connected 2-dimensional Riemannian manifold of constant sectional curvature equal to $k$. Let $\triangle(x, y, z) \subset Z$ be a geodesic triangle in $Z$ with vertices $x, y, z \in Z$. The angle of $\triangle(x, y, z)$ at vertex $x$, for example, will be denoted by $\measuredangle \triangle(x)$. A comparison triangle for $\triangle(x, y, z) \subset Z$ 
in $\mathbb{M}_{k}^{2}$ is a geodesic triangle $\bar{\triangle}_{k}(\bar{x}, \bar{y}, \bar{z}) \subset \mathbb{M}_{k}^{2}$ verifying

$$
d_{\mathbb{M}_{k}^{2}}(\bar{x}, \bar{y})=d_{Z}(x, y), \quad d_{\mathbb{M}_{k}^{2}}(\bar{y}, \bar{z})=d_{Z}(y, z), \quad \text { and } \quad d_{\mathbb{M}_{k}^{2}}(\bar{z}, \bar{x})=d_{Z}(z, x) .
$$

\section{DEFINITION 6}

A length space $Z$ is called an Alexandrov space of curvature not smaller than $k \in \mathbb{R}$ if every point of $Z$ has a neighborhood $U$ such that the angles of every triangle $\triangle(x, y, z) \subset U$ are well defined and satisfy the inequalities

$$
\measuredangle_{\triangle}(x) \geq \measuredangle_{\bar{\triangle}_{k}}(\bar{x}), \quad \measuredangle_{\triangle}(y) \geq \measuredangle_{\bar{\triangle}_{k}}(\bar{y}), \quad \text { and } \quad \measuredangle_{\triangle}(z) \geq \measuredangle_{\bar{\triangle}_{k}}(\bar{z})
$$

for every comparison triangle $\bar{\triangle}_{k}(\bar{x}, \bar{y}, \bar{z}) \subset \mathbb{M}_{k}^{2}$ of $\triangle$.

Suppose from now on that $Z$ is an $n$-dimensional Alexandrov space of curvature not smaller than $k \in \mathbb{R}$. Given three points $x, y, z \in Z$, let $\bar{\triangle}_{k}(\bar{x}, \bar{y}, \bar{z})$ be a triangle in $\mathbb{M}_{k}^{2}$ verifying

$$
d_{\mathbb{M}_{k}^{2}}(\bar{x}, \bar{y})=d_{Z}(x, y), \quad d_{\mathbb{M}_{k}^{2}}(\bar{y}, \bar{z})=d_{Z}(y, z), \quad \text { and } \quad d_{\mathbb{M}_{k}^{2}}(\bar{z}, \bar{x})=d_{Z}(z, x) .
$$

We denote by $\measuredangle_{k}(x ; y, z)$ the angle of $\bar{\triangle}_{k}(\bar{x}, \bar{y}, \bar{z})$ at $\bar{x}$. Note that this definition does not depend on the choice of the triangle $\bar{\triangle}_{k}(\bar{x}, \bar{y}, \bar{z})$.

Consider $z \in Z$ and $\lambda \in(0, \pi)$. The point $z$ is said to be $\lambda$-strained if there is a set $\left\{\left(a_{i}, b_{i}\right) \in Z \times Z ; i \in\{1, \ldots, n\}\right\}$, which is called a $\lambda$-strainer at $z$, such that $\measuredangle_{k}\left(z ; a_{i}, b_{i}\right)>\pi-\lambda$ and

$$
\max \left\{\left|\measuredangle_{k}\left(z ; a_{i}, a_{j}\right)-\frac{\pi}{2}\right|,\left|\measuredangle_{k}\left(z ; b_{i}, b_{j}\right)-\frac{\pi}{2}\right|,\left|\measuredangle_{k}\left(z ; a_{i}, b_{j}\right)-\frac{\pi}{2}\right|\right\}<\lambda
$$

for all $i \neq j \in\{1, \ldots, n\}$. The set $R_{\lambda}(Z)$ of $\lambda$-strained points of $Z$ is called the set of $\lambda$-regular points of $Z$. The points of $Z-R_{\lambda}(Z)$ are called $\lambda$-cone points of $Z$. It is a remarkable fact that $R_{\lambda}(Z)$ is an open and dense subset of $Z$.

We are interested in noncomplete Alexandrov spaces of dimension 2. It is well known (see $[\mathrm{BBI}]$ ) that these spaces are topological 2-manifolds possibly with boundary. With the previous definition, all boundary points of a noncomplete Alexandrov space of dimension 2 are cone points. To treat these spaces more accurately, we shall need the following definition.

Consider $\lambda \in(0, \pi)$ and a noncomplete Alexandrov space $Z$ of dimension 2. A point $z \in \partial Z$ is said to be $\lambda$-strained if there is a set $\{a, b, c\} \subset Z$, which is called a $\lambda$-strainer at $z$, such that $\measuredangle_{k}(z ; a, b)>\pi-\lambda$ and

$$
\max \left\{\left|\measuredangle_{k}(z ; a, c)-\frac{\pi}{2}\right|,\left|\measuredangle_{k}(z ; b, c)-\frac{\pi}{2}\right|\right\}<\lambda .
$$

The set $\partial R_{\lambda}(Z)$ of $\lambda$-strained points of $\partial Z$ is called the set of $\lambda$-regular points of $\partial Z$ or set of boundary $\lambda$-regular points of $Z$. The points of $\partial Z-\partial R_{\lambda}(Z)$ are called $\lambda$-cone points of $\partial Z$ or boundary $\lambda$-cone points of $Z$. When distinction is needed, the points of $R_{\lambda}(Z)$ (resp., of $Z-R_{\lambda}(Z)$ ) will be called interior $\lambda$-regular points of $Z$ (resp., interior $\lambda$-cone points of $Z$ ).

Recall now the notion of (pointed) Hausdorff-Gromov convergence. 


\section{DEFINITION 7}

Let $\left(Z_{i}, z_{i}\right)$ be a sequence of (pointed) metric spaces. We say that the sequence $\left(Z_{i}, z_{i}\right)$ converges in the (pointed) Hausdorff-Gromov sense to a (pointed) metric space $\left(Z, z_{0}\right)$ if the following holds: for every $r>\varepsilon>0$, there exist $i_{0} \in \mathbb{N}$ and a sequence of (maybe noncontinuous) maps $f_{i}: B_{Z_{i}}\left(z_{i}, r\right) \rightarrow Z\left(i>i_{0}\right)$ such that

(i) $f_{i}\left(z_{i}\right)=z_{0}$;

(ii) $\sup \left\{d_{Z^{\prime}}\left(f_{i}\left(z_{1}\right), f_{i}\left(z_{2}\right)\right)-d_{Z}\left(z_{1}, z_{2}\right) ; z_{1}, z_{2} \in Z\right\}<\varepsilon$; and

(iii) $B_{Z}\left(z_{0}, r-\varepsilon\right) \subset B_{Z}\left(f_{i}\left(B_{Z_{i}}\left(z_{i}, r\right)\right), \varepsilon\right)$.

Let us point out that, throughout the rest of the article, the term "converges" is going to stand for "converges in the (pointed) Hausdorff-Gromov sense."

Let $\left(Z_{i}, z_{i}\right)$ be a convergent sequence of Alexandrov spaces with the same lower curvature bound $k \in \mathbb{R}$ and the same dimension $n \in \mathbb{N}$. The limit Alexandrov space must have the same lower curvature bound $k$, but can have dimension less than or equal to $n$ (see [BBI, Corollary 10.8.25]). When the limit Alexandrov space has dimension $n$, Perelman's stability theorem (see [Kap]) ensures that it is homeomorphic to $Z_{i}$, for sufficiently large indices.

It is a fundamental fact that the class of Alexandrov spaces of curvature not smaller than $k \in \mathbb{R}$ is precompact with respect to the Hausdorff-Gromov convergence (see [Gro, Proposition 5.2], [BBI, Corollary 10.8.25]). More precisely, every sequence of pointed Alexandrov spaces of curvature not smaller than $k \in \mathbb{R}$ admits a convergent subsequence to an Alexandrov space with the same lower bound for the curvature.

The following result, due to Gromov (see [Gro, Theorem 8.30, Corollary 8.39]), will also be needed.

\section{THEOREM 8 (GROMOV)}

Let $\left(M_{i}, p_{i}\right)$ be a sequence of pointed complete Riemannian $n$-manifolds converging to a pointed Alexandrov space $\left(Z, z_{0}\right)$. Suppose there exists $\delta>0$ such that the sectional curvatures of $M_{i}$ are uniformly bounded on the balls $B_{M_{i}}\left(p_{i}, \delta\right)$. If the sequence $r_{\mathrm{inj}}^{M_{i}}\left(p_{i}\right)$ of the Riemannian injectivity radius of $p_{i}$ in $M_{i}$ converges to zero, then the dimension of $Z$ is strictly smaller than $n$.

Note that Theorem 8 is a local version of the Gromov theorem. The proof of [Gro, Theorem 8.30] is local and applies equally well to our hypotheses. We obtain that $z_{0}$ has a neighborhood $U$ in $Z$ that is isometric to the quotient of a Riemannian ball by a pseudogroup of its isometries with dimension almost 1 . Therefore (from the proof of Corollary 8.39), $U$ must have Hausdorff dimension strictly smaller than $n$. Since the dimension of an Alexandrov space is a well-defined integer (see [BBI, Theorem 10.8.2, Corollary 10.8.16]), Theorem 8 follows.

The fibration theorem is a useful tool for the study of sequences of Alexandrov spaces. The first version of this theorem, due to Fukaya (see [Fuk]), concerns Riemannian manifolds with pinched curvature. Later this theorem was extended by Takao Yamaguchi to Alexandrov spaces (see [Y2, Theorem 0.2], [SY], [Bel]). 
We would like to single out the fact that the statement of [Y2, Theorem 0.2] concerns complete Alexandrov spaces. However, the construction of the fibration is local and remains valid over compact domains (consisting of regular points) of the base Alexandrov space (see [Bar]). For the convenience of the reader, we present here the exact statement of the Yamaguchi theorem that will be needed in this article.

\section{THEOREM 9 (YAMAGUCHI)}

Let $\left(M_{i}, p_{i}\right)$ be a sequence of pointed complete Riemannian manifolds of dimension 3 with sectional curvature bounded from below by -1 . Suppose that the sequence $\left(M_{i}, p_{i}\right)$ converges to a pointed complete length space $(Z, z)$ of dimension 2 (resp., dimension 1). Then there exists a constant $\lambda>0$ satisfying the following condition: for every compact domain $Y$ of $Z$ contained in $R_{\lambda}(Z)$ and, for sufficiently large $i_{0}=i_{0}(Y) \in \mathbb{N}$, there exist:

(i) a sequence $\tau_{i}>0\left(i>i_{0}\right)$ converging to zero;

(ii) a sequence $\mathcal{N}_{i}\left(i>i_{0}\right)$ of compact 3-submanifolds of $M_{i}$ (perhaps with boundary); and

(iii) a sequence of $\tau_{i}$-approximations $\mathfrak{p}: \mathcal{N}_{i} \rightarrow Y$ which induces a structure of locally trivial fiber bundle on $\mathcal{N}_{i}$. Furthermore, the fibers of this fibration are circles (resp., spheres, tori).

\section{Sequences of hyperbolic cone manifolds}

Let $M$ be a closed, orientable, and irreducible differential manifold of dimension 3, and let $\Sigma=\Sigma_{1} \sqcup \cdots \sqcup \Sigma_{l}$ be an embedded link in $M$. A hyperbolic cone structure with topological type $(M, \Sigma)$ is a complete intrinsic metric on $M$ such that every nonsingular point (i.e., every point in $M-\Sigma$ ) has a neighborhood isometric to an open set of $\mathbb{H}^{3}$, the hyperbolic space of dimension 3 , and such that every singular point (i.e., every point in $\Sigma$ ) has a neighborhood isometric to an open neighborhood of a singular point of $\mathbb{H}^{3}(\alpha)$, the space obtained by identifying the sides of a wedge of angle $\alpha \in(0,2 \pi]$ in $\mathbb{H}^{3}$ by a rotation about the axis of the wedge (see Figure 1). The angles $\alpha$ are called cone angles and they may vary from one connected component of $\Sigma$ to another. Conventionally, the complete structure $M_{0}$ on $M-\Sigma$ (see [Koj]) is considered as a hyperbolic cone structure with topological type $(M, \Sigma)$ and cone angles equal to zero.

We point out (see [BLP, Proposition 3.3]) that every hyperbolic cone manifold is an Alexandrov space of curvature bounded from below by -1 .

A natural way to study degenerated deformations of hyperbolic cone structures on $(M, \Sigma)$ is to consider sequences of hyperbolic cone structures converging to the limit Alexandrov space. To study this type of sequence, we need the important notion of collapsing which illustrates the intuitive fact that the volume of the sequence may or may not be reduced to zero. 


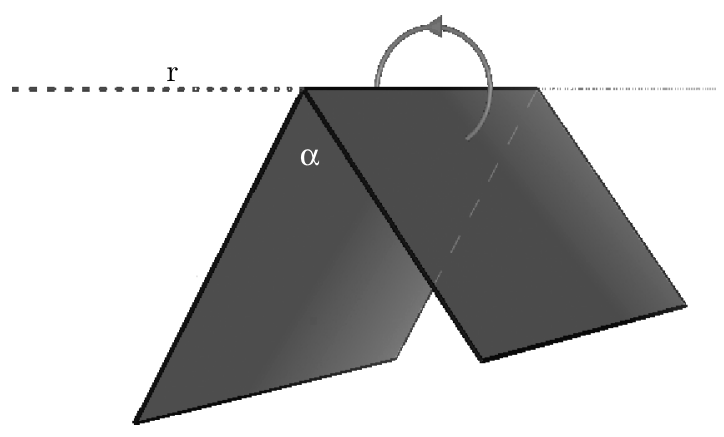

Figure 1

\section{DEFINITION 10}

We say that a sequence $M_{i}$ of hyperbolic cone manifolds with topological type $(M, \Sigma)$ collapses if, for every sequence of points $p_{i} \in M-\Sigma$, the sequence $r_{\mathrm{inj}}^{M_{i}-\Sigma}\left(p_{i}\right)$ consisting of their Riemannian injectivity radii in $M_{i}-\Sigma$ converges to zero. Otherwise, we say that the sequence $M_{i}$ does not collapse.

Given a sequence $M_{i}$ of hyperbolic cone manifolds with topological type $(M, \Sigma)$, fix indices $i \in \mathbb{N}$ and $j \in\{1, \ldots, l\}$. For sufficiently small radius $R>0$, the metric neighborhood

$$
B_{M_{i}}\left(\Sigma_{j}, R\right)=\left\{x \in M_{i} ; d_{M_{i}}\left(x, \Sigma_{j}\right)<R\right\}
$$

of $\Sigma$ is a solid torus embedded in $M_{i}$. The supremum of the radius $R>0$ satisfying the above property is called the normal injectivity radius of $\Sigma_{j}$ in $M_{i}$ and we denote it by $R_{i}\left(\Sigma_{j}\right)$. Analogously we can define $R_{i}(\Sigma)$, the normal injectivity radius of $\Sigma$. It is a remarkable fact (see [Fuj] and [HK]) that the existence of a uniform lower bound for $R_{i}(\Sigma)$ ensures the existence of a sequence of points $p_{i_{k}} \in M$ such that the sequence $\left(M_{i_{k}}, p_{i_{k}}\right)$ converges to a pointed hyperbolic cone manifold $\left(M_{\infty}, p_{\infty}\right)$ with topological type $(M, \Sigma)$. Moreover, $M_{\infty}$ must be compact provided that the cone angles of $M_{i_{k}}$ are uniformly bounded from below.

This section is divided into three parts. The first part contains some preliminary results, the second part presents the proof of Theorem 2, and the last one concerns some applications (including Corollaries 4, 5). Let us point out that, throughout the rest of the article, the term "component" is going to stand for "connected component."

\subsection{Preliminary results}

Let us begin with three elementary lemmas which will be important for the proof of Theorem 2.

LEMMA 11

Suppose that $M-\Sigma$ is hyperbolic, and let $T$ be a 2-dimensional torus embedded 
in $M-\Sigma$. Then $T$ separates $M$. Moreover, one and only one of the following statements holds:

(i) $T$ is parallel to a component of $\Sigma$ (hence, it bounds a solid torus in $M$ );

(ii) $T$ is not parallel to a component of $\Sigma$ and it bounds a solid torus in $M-\Sigma$;

(iii) $T$ is not parallel to a component of $\Sigma$ and it is contained in a ball $B$ of $M-\Sigma$. Furthermore, $T$ bounds a region in $B$ which is homeomorphic to the exterior of a knot in $S^{3}$.

Proof

Suppose that $T$ is not parallel to a connected component of $\Sigma$. Since $M-\Sigma$ is hyperbolic, $T$ is compressible in $M-\Sigma$, and hence, it splits $M$ into two components, say, $U$ and $V$. Denote by $\Sigma_{U}$ and by $\Sigma_{V}$ the subsets of $\Sigma$ contained in $U$ and in $V$, respectively. Without loss of generality, suppose that $\Sigma_{U} \neq \emptyset$.

Given a compression disk $D$ for $T$, let $S$ be the sphere obtained by the compression of $T$ along $D$. Since $M-\Sigma$ is irreducible and $\Sigma_{U} \neq \emptyset$, the sphere $S$ separates $M-\Sigma$ (and therefore separates $M$ ) and bounds a ball $B \subset M-\Sigma$ in the opposite side of $\Sigma_{U}$. In particular, this implies that $\Sigma_{V}=\emptyset$, since $\Sigma_{U}$ and $\Sigma_{V}$ lie in different sides of $S$, by construction. Thus, $V$ must satisfy one of the assertions (ii) or (iii) according to whether $D \subset V$ or $D \subset U$.

\section{LEMMA 12}

Given a closed ball $B \subset M-\Sigma$ and an embedded 2-dimensional torus $T \subset B$, let $U$ and $W$ be the compact three manifolds (with toral boundary) obtained by cutting $M$ along $T$. (Suppose that $W \subset B$.) If $W$ is homeomorphic to the exterior of a knot in $S^{3}$, then we can replace $W$ by a solid torus $V$ without changing the topological type of $M$.

\section{Proof}

Let $B^{\prime}$ be a closed ball, and let $h: \partial B \rightarrow \partial B^{\prime}$ be a diffeomorphism such that $B \cup_{h} B^{\prime}$, the manifold obtained by gluing $B$ and $B^{\prime}$ through $h$, is diffeomorphic to $S^{3}$. Note that the manifold $V^{\prime}=(U \cap B) \cup_{h} B^{\prime}$ is a closed solid torus. Let $V$ be a closed solid torus, and let $g: \partial V \rightarrow \partial V^{\prime}$ be a diffeomorphism such that $V \cup_{g} V^{\prime}$, the manifold obtained by gluing $V$ and $V^{\prime}$ through $g$, is diffeomorphic to $S^{3}$. Thus, the compact 3-manifold $\mathcal{B}=\left(V^{\prime} \cup_{g} V\right)-\operatorname{int}\left(B^{\prime}\right)=(U \cap B) \cup_{g} V$ is diffeomorphic to a closed ball. It is a standard result of low-dimensional topology that the manifold

$$
(M-\operatorname{int}(B)) \cup_{\text {Id }} \mathcal{B}=(M-W) \cup_{g} V,
$$

obtained using Id $: \partial B \rightarrow \partial B$ as the gluing map, is diffeomorphic to $M$.

\section{LEMMA 13}

Consider $\varepsilon \in(0,1)$, and let $\mathcal{M}$ be a hyperbolic cone manifold with topological type $(M, \Sigma)$ together with a metric neighborhood $\mathcal{B}$ of $\Sigma$ whose components are 
homeomorphic to solid tori. Denote by $g$ the hyperbolic metric on $\mathcal{M}-\Sigma$, and let $h$ be a Riemannian metric on $M$ such that

(i) $h$ coincides with $g$ on $\mathcal{M}-\mathcal{B}$;

(ii) $h$ is an $\varepsilon$-perturbation of $g$ on $M-\Sigma$, that is,

$$
\left|\|v\|_{g}-\|v\|_{h}\right|<\varepsilon
$$

for every $x \in \mathcal{M}-\Sigma$ and $v \in K_{x}=\left\{u \in T_{x}(\mathcal{M}-\Sigma) ;\|u\|_{g} \leq 2\right\}$.

Then the Hausdorff-Gromov distance between $\mathcal{M}$ and the Riemannian manifold $\mathcal{N}=(M, h)$ is smaller than $4 \varepsilon D$, where $D=1+\operatorname{diam}_{\mathcal{M}}(M)$.

\section{Proof}

To prove the lemma, it suffices to check that the identity map Id $: \mathcal{M} \rightarrow \mathcal{N}$ is a $2 \varepsilon D$-isometry. Fix $x, y \in M$. Since $\Sigma$ has codimension 2 , there is a differentiable curve $\alpha:[0, L] \rightarrow \mathcal{M}$ (parameterized by arc length in $\mathcal{M}$ ) such that $\alpha((0, L)) \subset$ $\mathcal{M}-\Sigma$ and $L<d_{\mathcal{M}}(x, y)+\frac{\varepsilon}{2}$. Then

$$
\begin{aligned}
d_{\mathcal{N}}(x, y)-d_{\mathcal{M}}(x, y) & \leq \mathcal{L}_{\mathcal{N}}(\alpha)-\mathcal{L}_{\mathcal{M}}(\alpha)+\frac{\varepsilon}{2} \\
& \leq \int_{0}^{L}\left|\left\|\alpha^{\prime}(t)\right\|_{g}-\left\|\alpha^{\prime}(t)\right\|_{h}\right| d t+\frac{\varepsilon}{2} \\
& <\varepsilon \cdot d_{\mathcal{M}}(x, y)+\frac{\varepsilon^{2}}{2}+\frac{\varepsilon}{2} \leq \varepsilon D,
\end{aligned}
$$

and in particular, $\operatorname{diam}_{\mathcal{N}}(M) \leq 2 D$. Similarly, there is a differentiable curve $\beta$ : $\left[0, L^{\prime}\right] \rightarrow \mathcal{N}$ (parameterized by arc length in $\left.\mathcal{N}\right)$ such that $\beta\left(\left(0, L^{\prime}\right)\right) \subset \mathcal{N}-\Sigma$ and $L^{\prime}<d_{\mathcal{N}}(x, y)+\frac{\varepsilon}{2}$. Inequality (3) implies that

$$
\left\{u \in T_{z}(\mathcal{M}-\Sigma) ; h_{z}(u, u)=1\right\} \subset K_{z},
$$

for all $z \in \mathcal{N}-\Sigma$. So

$$
\left\|\beta^{\prime}(t)\right\|_{g}-\left\|\beta^{\prime}(t)\right\|_{h} \mid<\varepsilon,
$$

for all $t \in\left[0, L^{\prime}\right]$. Analogously to the preceding case, it follows that $d_{\mathcal{M}}(x, y)-$ $d_{\mathcal{N}}(x, y) \leq 2 \varepsilon D$.

\subsection{Proof of Theorem 2}

The purpose of this section is to study a collapsing sequence $M_{i}$. According to [Fuj, Theorem 1], the sequence $M_{i}$ cannot collapse when $\lim _{i \rightarrow \infty} R_{i}\left(\Sigma_{j}\right)=\infty$ for all components $\Sigma_{j}$ of $\Sigma$. Given $p \in \Sigma_{1}$, we can assume without loss of generality that $\sup \left\{R_{i}\left(\Sigma_{1}\right) ; i \in \mathbb{N}\right\}<\infty$ and that the sequence $\left(M_{i}, p\right)$ converges to a pointed Alexandrov space $\left(Z, z_{0}\right)$. We are interested in the case where the length of the singularity remains uniformly bounded, that is, where

$$
\sup \left\{\mathcal{L}_{M_{i}}\left(\Sigma_{j}\right) ; i \in \mathbb{N}, j \in\{1, \ldots, l\}\right\}<\infty .
$$

Passing to a subsequence if necessary, we can suppose that each component $\Sigma_{j}$ of $\Sigma$ satisfies one and only one of the following statements: 
1. $\sup \left\{d_{M_{i}}\left(p_{i}, \Sigma_{j}\right) ; i \in \mathbb{N}\right\}<\infty$ and $\Sigma_{j}$ converges to a closed curve $\Sigma_{j}^{Z} \subset Z$,

2. $\lim _{i \in \mathbb{N}} d_{M_{i}}\left(p_{i}, \Sigma_{j}\right)=\infty$.

This dichotomy allows us to write $\Sigma=\Sigma_{0} \sqcup \Sigma_{\infty}$, where $\Sigma_{0}$ contains the components $\Sigma_{j}$ of $\Sigma$ which satisfy item (1) (in particular, $\Sigma_{1} \subset \Sigma_{0}$ ) and $\Sigma_{\infty}$ contains those which satisfy item (2). Now consider the compact set

$$
\Sigma_{Z}=\bigcup_{\Sigma_{j} \subset \Sigma_{0}} \Sigma_{j}^{Z} \subset Z
$$

The following proposition provides information on the dimension of the limit of a sequence of hyperbolic cone manifolds which collapses.

\section{LEMMA 14}

Given a sequence $p_{i} \in M$, suppose that the sequence $\left(M_{i}, p_{i}\right)$ converges to a pointed Alexandrov space $\left(Z, z_{0}\right)$. If the sequence $M_{i}$ collapses and $\Sigma_{Z}$ is not dense in $Z$, then the dimension of $Z$ is strictly smaller than 3 .

Proof

Since $\Sigma_{Z}$ is not dense in $Z$, there is $z_{\infty} \in Z-\Sigma_{Z}$ such that $\delta=d_{Z}\left(z_{\infty}, \Sigma_{Z}\right)>0$. Take a sequence $q_{i} \in M-\Sigma$ such that

$$
\lim _{i \rightarrow \infty} q_{i}=z_{\infty} \quad \text { and } \quad B_{M_{i}}\left(q_{i}, \frac{\delta}{3}\right) \cap B_{M_{i}}\left(\Sigma, \frac{\delta}{3}\right)=\emptyset .
$$

By definition, the sequence $\left(M_{i}, q_{i}\right)$ converges to $\left(Z, z_{\infty}\right)$. Since the sequence $M_{i}$ collapses, we have $\lim _{i \rightarrow \infty} r_{\text {inj }}^{M_{i}}\left(q_{i}\right)=0$. According to [Sal, proof of Proposition 4], we can change the metrics of $M_{i}$ in neighborhoods of $\Sigma$ contained in $B_{M_{i}}\left(\Sigma, \frac{\delta}{3}\right)$ to obtain a sequence of complete Riemannian manifolds $N_{i}$ homeomorphic to $M$ which have finite volume and sectional curvature bounded from below by -1 . Furthermore, thanks to Lemma 13, we can suppose that the sequence $\left(N_{i}, q_{i}\right)$ converges to $\left(Z, z_{0}\right)$. Note that, by construction, the balls $B_{N_{i}}\left(q_{i}, \frac{\delta}{3}\right)$ and $B_{M_{i}}\left(q_{i}, \frac{\delta}{3}\right)$ are isometric. Then we have $\lim _{i \rightarrow \infty} r_{\text {inj }}^{N_{i}}\left(q_{i}\right)=0$. According to Theorem 8 , there is $\lambda \in\left(0, \frac{\delta}{3}\right)$ such that the ball $B_{Z}\left(z_{\infty}, \lambda\right)$ has Hausdorff dimension strictly smaller than 3. Since the dimension of an Alexandrov space is a well-defined integer, it follows that $Z$ also has dimension strictly smaller than 3 .

The preceding lemma says that the unique possibilities for the dimension of $Z$ are 2,1 , or 0 . The proof of Theorem 2 will be split according to these three cases.

\subsubsection{Case where $Z$ has dimension 2}

It is a classical result of metric geometry that all 2-dimensional Alexandrov spaces are topological manifolds of dimension 2, possibly with boundary. We can describe the boundary of $Z$ as

$$
\partial Z=\bigsqcup_{k \in \Gamma} \partial_{k} Z,
$$


where the $\partial_{k} Z$ 's are the connected components of $\partial Z$. Note that each component $\partial_{k} Z$ is homeomorphic to a circle or to a straight line.

\section{THEOREM 15 (COLLAPSING: DIMENSION 2)}

Suppose that there is a point in $p \in \Sigma_{1}$ such that the sequence $\left(M_{i}, p\right)$ converges to a 2-dimensional pointed Alexandrov space $\left(Z, z_{0}\right)$. If $\sup \left\{R_{i}\left(\Sigma_{1}\right) ; i \in \mathbb{N}\right\}<\infty$ and

$$
\sup \left\{\mathcal{L}_{M_{i}}\left(\Sigma_{j}\right) ; i \in \mathbb{N}, j \in\{1, \ldots, l\}\right\}<\infty,
$$

then it follows that

(i) $M$ is Seifert fibered;

(ii) if $\partial Z \neq \emptyset$, then $\partial Z$ has only one connected component and $M$ is a lens space;

(iii) if $Z$ is not compact, then $\lim _{i \in \mathbb{N}} R_{i}\left(\Sigma_{j}\right)=\infty$ for any component $\Sigma_{j}$ of $\Sigma_{\infty}$.

\section{Proof}

According to [Sal, proof of Proposition 4], the metric of hyperbolic cone manifolds $M_{i}$ can be deformed, in some small neighborhoods of $\Sigma$, to yield a sequence of complete Riemannian manifolds $N_{i}$ (homeomorphic to $M$ ) with sectional curvature not smaller than -1 . Furthermore, thanks to Lemma 13, we can suppose that the sequence $\left(N_{i}, p\right)$ converges to $\left(Z, z_{0}\right)$.

If $Z$ is compact, then the statement follows directly from the results of Shioya and Yamaguchi [SY]. In fact, when $Z$ does not have boundary, [SY, Theorem 0.2] can be applied to the sequence $N_{i}$ to conclude that $M$ is Seifert fibered. When $Z$ has boundary, [SY, Corollary 0.4] can be applied to the sequence $N_{i}$ to conclude ( $M$ is irreducible) that $Z$ is a closed disk with at most one cone point in its interior. In this case, [SY, Theorem 0.3] implies that $M$ is a lens space. (In particular, it is also Seifert fibered.)

Suppose from now on that $Z$ is not compact. Since the normal injectivity radii of components of $\Sigma_{0}$ are uniformly bounded, there is $R>0$ such that

$$
B_{M_{i}}\left(\Sigma_{j}, R_{i}\left(\Sigma_{j}\right)\right) \subset B_{M_{i}}(p, R / 2)
$$

for all $i \in \mathbb{N}$ and for every component $\Sigma_{j} \subset \Sigma_{0}$. Let $K$ be a compact and connected 2-dimensional submanifold of $Z$ such that $B_{Z}\left(z_{0}, R\right) \subset K$ (therefore $\Sigma_{Z} \subset K$ ), $\partial K$ is a disjoint union of circles, and $Z-K$ is a disjoint union of components of infinite diameter. Set $\Lambda=\left\{k \in \Gamma ; \partial_{k} Z \cap K \neq \emptyset\right\}$. If $\partial Z \neq \emptyset$, then we assume also that:

- $\Lambda \neq \emptyset$, that is, $K \cap \partial Z \neq \emptyset$,

- $\partial_{k} Z \cap K=\partial_{k} Z$ for all $k \in \Lambda$ such that the component $\partial_{k} Z$ is compact,

- $\partial_{k} Z \cap K$ is connected for all $k \in \Lambda$. 


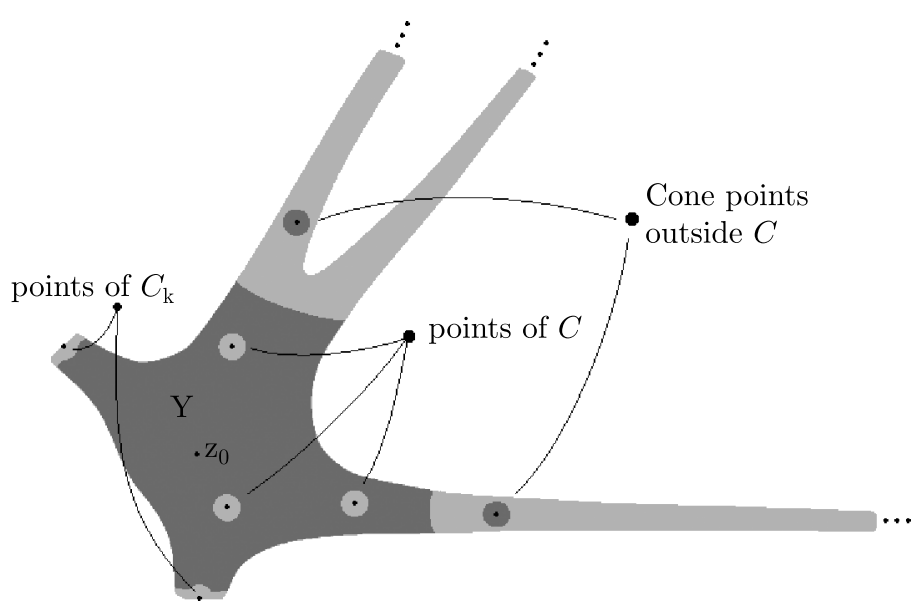

Figure 2. Compact $Y$ and the sets $\mathcal{C}$ and $\mathcal{C}_{k}$ of cone points.

For each $k \in \Lambda$, denote by $\partial_{k} K$ the (unique by construction) connected component of $\partial K$ such that $\partial_{k} Z \cap \partial_{k} K \neq \emptyset$. The boundary of $K$ is given by

$$
\partial K=\bigsqcup_{k \in \Lambda} \partial_{k} K \sqcup \bigsqcup_{m \in \Gamma-\Lambda} \partial_{m} K,
$$

where the $\partial_{m} K$ 's are the components of $\partial K$ which do not intersect the boundary of $Z$. Let $\lambda$ be the constant given by the fibration theorem (Theorem 9). Since $K$ is compact, the sets of $\lambda$-cone points

$$
\begin{aligned}
\mathcal{C} & =\left[Z-R_{\lambda}(Z)\right] \cap K, \\
\mathcal{C}_{k} & =\left[\partial_{k} Z-\partial R_{\lambda}(Z)\right] \cap K \subset \partial_{k} K \quad(k \in \Lambda)
\end{aligned}
$$

are finite (see [SY, Theorem 2.1]). Modulo increasing $K$, we can assume that $C$ is contained in its interior. Consider $s_{1}>s_{2}>0$ verifying

- $B_{K}\left[z, s_{1}\right] \subset \operatorname{int}(K)$ and $B_{K}\left[z, s_{1}\right]$ is homeomorphic to $D^{2}$ for all $z \in \mathcal{C}$,

- $B_{K}\left[z, s_{1}\right] \cap B_{K}\left[z^{\prime}, s_{1}\right]=\emptyset$ for all $z, z^{\prime} \in \mathcal{C} \cup \bigcup_{k \in \Lambda} \mathcal{C}_{k}$,

- for all $z \in \mathcal{C} \cup \bigcup_{k \in \Lambda} \mathcal{C}_{k}$ and for all $k \in \Lambda$, we have

$$
B_{K}\left(\partial_{k} K, s_{2}\right) \cap B_{K}\left(z, s_{1}\right) \neq \emptyset \quad \text { if and only if } \quad z \in \mathcal{C}_{k} .
$$

Let $Y$ be the compact subset of $Z$ defined by

$$
Y=K-\bigcup_{z \in \mathcal{C}} B_{K}\left(z, s_{1}\right)-\bigsqcup_{k \in \Lambda} U_{k} \subset R_{\lambda}(Z),
$$

where $U_{k}=B_{K}\left(\partial_{k} Z, s_{2}\right) \cup \bigcup_{z \in \mathcal{C}_{k}} B_{K}\left(z, s_{1}\right)$ for every $k \in \Lambda$. The boundary of $Y$ is given by

$$
\partial Y=\bigsqcup_{k \in \Lambda} \partial_{k} Y \sqcup \bigsqcup_{z \in \mathcal{C}} \partial_{z} Y \sqcup \bigsqcup_{m \in \Gamma-\Lambda} \partial_{m} K
$$

where the $\partial_{k} Y$ 's and $\partial_{z} Y^{\prime}$ 's are the components of $\partial Y$ which intersect the regions $U_{k}$ and $B_{K}\left(z, s_{1}\right)$, respectively (see Figure 2). Without loss of generality, the 

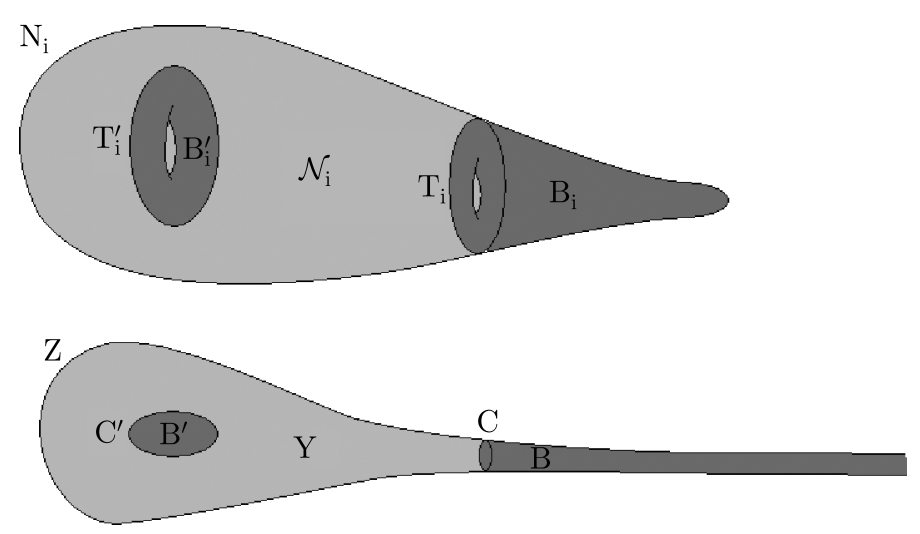

Figure 3

fibration theorem (Theorem 9) gives us a sequence $\mathcal{N}_{i}$ of compact and connected 3-dimensional submanifolds of $M$, a sequence $\tau_{i}>0$ that converges to zero, and a sequence of $\tau_{i}$-approximations $\mathfrak{p}_{i}: \mathcal{N}_{i} \rightarrow Y$ which induces a structure of locally trivial circle bundle on $\mathcal{N}_{i}$. Since the manifold $M$ is orientable, it follows that the connected components of $\partial \mathcal{N}_{i}=\mathfrak{p}_{i}^{-1}(\partial Y)$ are 2-dimensional tori. Let $C$ be a component of $\partial Y$, and let $T_{i}$ be the component of $\partial \mathcal{N}_{i}$ associated with it $\left(T_{i}=\right.$ $\mathfrak{p}_{i}^{-1}(C)$ ). By construction (see Figure 3 ), we can choose base points $q_{i} \in T_{i}$ and $q \in C$ such that the pointed components $\left(B_{i}, q_{i}\right)$ of $N_{i}-\operatorname{int}\left(\mathcal{N}_{i}\right)$ associated with the torus $T_{i}\left(\partial B_{i}=T_{i}\right)$ converge to the pointed component $(B, q)$ of $Z-\operatorname{int}(Y)$ associated to $C(\partial B=C)$.

\section{LEMMA 16}

There exists $i_{0} \in N$ such that, for every $i>i_{0}$, the components of $N_{i}-\mathcal{N}_{i}$ are homeomorphic to either a solid torus or to the exterior of a knot in $S^{3}$ which, in addition, is contained in an embedded ball in $M-\Sigma$.

Proof

A boundary torus of $N_{i}-\mathcal{N}_{i}$ has three possible natures according to the decomposition of $\partial Y$ from (4). We shall consider separately each of these possibilities.

First type: The torus $T_{i}^{z}=\mathfrak{p}_{i}^{-1}\left(\partial_{z} Y\right) \subset \partial N_{i}, z \in \mathcal{C}$. Consider a point $z \in \mathcal{C}$. By rescaling the metric with respect to the length of the fibers and using the stability theorem of Perelman (see [Kap]), Shioya and Yamaguchi [SY, Theorem 0.2] show that the region $B_{i}^{z}$ of $N_{i}-\mathcal{N}_{i}$ bounded by the torus $T_{i}^{z}$ is a solid torus for sufficiently large $i$. Furthermore, the solid torus $B_{i}^{z}$ is glued on $\mathcal{N}_{i}$ without "killing" the fiber.

Second type: The torus $T_{i}^{k}=\mathfrak{p}_{i}^{-1}\left(\partial_{k} Y\right) \subset \partial N_{i}, k \in \Lambda$. Given an index $k \in \Lambda$, we can decompose the component $\partial_{k} Y$ into two simple $\operatorname{arcs} \alpha$ and $\beta$ with the same ends, say, $P$ and $Q$, such that $\alpha=U_{k} \cap \partial_{k} Y$. Note that $\alpha=\partial_{k} Y$ and $\beta$ reduces to a single point (in particular, $P=Q$ ) when $\partial_{k} Z$ is compact. Let $B_{i}^{k}$ be the region of $N_{i}-\mathcal{N}_{i}$ bounded by the torus $T_{i}^{k}$. By using the same techniques, 


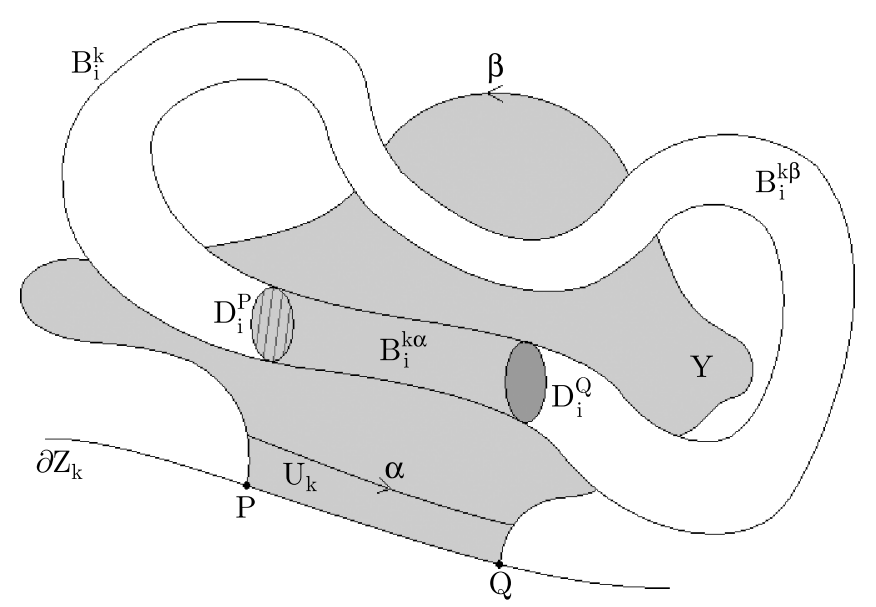

Figure 4. Tori of second type.

[SY, Theorem 0.3] shows that we can choose, for sufficiently large $i$, a region $B_{i}^{k \alpha}$ inside $B_{i}^{k}$ which is homeomorphic to $\alpha \times D^{2}$ (see Figure 4). Moreover,

- the sequence formed by the regions $B_{i}^{k \alpha}$ converges to the region $U_{k}$,

- the circle $\{z\} \times \partial D^{2}$ is a fiber of the Seifert fibration on the boundary of $\mathcal{N}_{i}$, for every $z \in \alpha$.

If $\partial_{k} Z$ is compact, then the regions $B_{i}^{k}$ are homeomorphic to solid tori for sufficiently large $i$. Suppose from now on that $\partial_{k} Z$ is not compact, and let $D_{i}^{P}$ and $D_{i}^{Q}$ be the disks of $B_{i}^{k \alpha}$ which are glued on the fibers $\mathfrak{p}_{i}^{-1}(P)$ and $\mathfrak{p}_{i}^{-1}(Q)$, respectively. The fibers above $\beta$ together with the disks $D_{i}^{P}$ and $D_{i}^{Q}$ yield an embedded sphere in $N_{i}-\Sigma$. This sphere splits $N_{i}$ into two regions: the region $B_{i}^{k \beta}=B_{i}^{k}-\operatorname{int}\left(B_{i}^{k \alpha}\right)$ and the region $N_{i}-\operatorname{int}\left(B_{i}^{k \beta}\right)$ which contains the singular set $\Sigma_{0} \neq \emptyset$. Since the manifold $N_{i}-\Sigma$ is irreducible, the region $B_{i}^{k \beta}$ must be homeomorphic to a ball, and therefore, for sufficiently large $i$, the region $B_{i}^{k}=$ $B_{i}^{k \alpha} \cup B_{i}^{k \beta}$ is homeomorphic to a solid torus.

Third type: The torus $T_{i}^{m}=\mathfrak{p}_{i}^{-1}\left(\partial_{m} K\right) \subset \partial N_{i}, m \in \Gamma-\Lambda$. Given an index $m \in \Gamma-\Lambda$, let $B_{i}^{m}$ be the region of $N_{i}-\mathcal{N}_{i}$ bounded by the torus $T_{i}^{k}$. Since $B_{Z}\left(z_{0}, R\right) \subset K$, the torus $T_{i}^{m}$ is contained in $M-\Sigma$ and cannot be parallel to a component of $\Sigma_{0}$. According to Lemma 11, the region $B_{i}^{m}$ is homeomorphic either to a solid torus (possibly having a component of $\Sigma_{\infty}$ as its soul) or to the exterior of a knot in $S^{3}$ which is contained in an embedded ball in $M-\Sigma$.

Fix from now on an index $i>i_{0}$. According to Lemma 12, the preceding claim shows that all components of $N_{i}-\mathcal{N}_{i}$ can be supposed to be homeomorphic to a solid torus. Let $\mathcal{W}_{i}$ be the 3-manifold which has been obtained by the union of $\mathcal{N}_{i}$ with all torus components of $N_{i}-\mathcal{N}_{i}$ which are glued to the boundary of $\mathcal{N}_{i}$ without "killing" the fibers of the Seifert fibration. For example, $B_{i}^{z} \subset \mathcal{W}_{i}$ and $B_{i}^{k} \subset N_{i}-\mathcal{W}_{i}$, for all $z \in \mathcal{C}$ and $k \in \Lambda$. The Seifert fibration on $\mathcal{N}_{i}$ (with base $Y$ ) 
naturally extends to a Seifert fibration on all of $\mathcal{W}_{i}$. Moreover, the underlying space of its basis is the topological surface $\mathcal{Y}$ which has been obtained by gluing disks on the components of $\partial Y$ which are associated with the components of $\mathcal{W}_{i}-\mathcal{N}_{i}$. If $\mathcal{W}_{i}=N_{i}$ (so that $\partial \mathcal{Y}=\emptyset$ ), then the manifold $M$ will be Seifert fibered and $\partial Z=\emptyset$. Thus, let us assume from now on that $\mathcal{W}_{i} \neq N_{i}$ (so that $\partial \mathcal{Y} \neq \emptyset$ and $\left.\partial \mathcal{W}_{i} \neq \emptyset\right)$. Suppose that there exists an essential arc $\gamma$ properly immersed in $\mathcal{Y}$. Then the fibration above $\gamma$ provides an essential annulus embedded in $\mathcal{W}_{i}$. Since all the components of $N_{i}-\mathcal{W}_{i}$ are solid tori which are glued to $\partial \mathcal{W}_{i}$ in order to "kill" the fibers of the Seifert fibration, this essential annulus becomes an essential sphere in $N_{i}$. This is impossible, since $M$ is irreducible. Therefore, $\mathcal{Y}$ has nonnegative Euler characteristic, it has exactly one boundary component, and it contains at most one interior cone point. In particular, it follows that $Z$ has at most one boundary component and at most one interior cone point. In fact, if $Z$ has more than one interior cone point (resp., boundary component), then the compact $K$ can be chosen containing two interior cone points (resp., two boundary components). This implies the existence of a second interior cone point (resp., boundary component) in $\mathcal{Y}$, which is a contradiction.

Because $\mathcal{Y}$ is compact, the above discussion implies that $\mathcal{Y}$ is homeomorphic to a disk $D^{2}$. So $\mathcal{W}_{i}$ is homeomorphic to a solid torus and this implies that $M$ is a lens space.

\subsubsection{Case where $Z$ has dimension 1}

It is also a standard result of metric geometry that all 1-dimensional Alexandrov spaces are topological manifolds of dimension 1. Precisely, they are homeomorphic to one of the following models: $S^{1},[0,1],[0, \infty)$, or $(-\infty, \infty)$.

\section{THEOREM 17 (COLLAPSING: DIMENSION 1)}

Suppose there is a point $p \in \Sigma_{1}$ such that the sequence $\left(M_{i}, p\right)$ converges to a 1-dimensional pointed Alexandrov space $\left(Z, z_{0}\right)$. If $\sup \left\{R_{i}\left(\Sigma_{1}\right) ; i \in \mathbb{N}\right\}<\infty$ and

$$
\sup \left\{\mathcal{L}_{M_{i}}\left(\Sigma_{j}\right) ; i \in \mathbb{N}, j \in\{1, \ldots, l\}\right\}<\infty,
$$

then it follows that

(i) $Z$ is homeomorphic to $S^{1}$ and $M$ is a Euclidean, Nil, or Sol manifold;

(ii) $Z$ is homeomorphic to $[0,1]$ and $M$ is a lens space or a Euclidean, Nil, Sol, or prism manifold;

(iii) $Z$ is homeomorphic to $[0, \infty)$ or $(-\infty, \infty), M$ is a lens space or prism manifold, and $\lim _{i \in \mathbb{N}} R_{i}\left(\Sigma_{j}\right)=\infty$, for every component $\Sigma_{j}$ of $\Sigma_{\infty}$.

\section{Proof}

If $Z$ is compact (i.e., homeomorphic to $S^{1}$ or $[0,1]$ ), then the assertions above follow directly from the results of Shioya and Yamaguchi [SY]. To be more precise, when $Z$ is homeomorphic to $S^{1}$, the irreducibility of $M$ implies that it is a Euclidean, Nil, or Sol manifold (see [SY, remark before Theorem 0.5 and Table 1]). When $Z$ is homeomorphic to [0,1], the irreducibility of $M$ implies 
that it is a lens space or a Euclidean, Nil, Sol, or prism manifold (see [SY, Table 1]).

Suppose from now on that $Z$ is not compact, that is, that $Z$ is homeomorphic to $[0, \infty)$ or $(-\infty, \infty)$. According to [Sal, proof of Proposition 4], the metric of hyperbolic cone manifolds $M_{i}$ on some small neighborhoods of $\Sigma$ can be deformed to yield a sequence of complete Riemannian manifolds $N_{i}$ (homeomorphic to $M$ ) with sectional curvature not smaller than -1 . Furthermore, thanks to Lemma 13, we can suppose that the sequence $\left(N_{i}, p\right)$ converges to $\left(Z, z_{0}\right)$. Since $\sup \left\{R_{i}\left(\Sigma_{j}\right) ; i \in \mathbb{N}, \Sigma_{j} \in \Sigma_{0}\right\}<\infty$, there is $R>0$ such that

$$
B_{M_{i}}\left(\Sigma_{j}, R_{i}\left(\Sigma_{j}\right)\right) \subset B_{M_{i}}(p, R / 2)
$$

for all $i \in \mathbb{N}$ and for every component $\Sigma_{j}$ of $\Sigma_{0}$. Consider a compact $K$ of $Z$ which is homeomorphic to $[0,1]$ and contains $B_{Z}\left(z_{0}, R\right) \cup \partial Z$. Let $\lambda$ be the constant given by the fibration theorem (Theorem 9), and set $Y=K-U$, where $U$ is empty or a small open neighborhood of $\partial Z$ according to whether or not the boundary of $Z$ is empty. Without loss of generality, the fibration theorem (Theorem 9) gives us a sequence $\mathcal{N}_{i}$ of compact and connected 3-dimensional submanifolds of $M$, a sequence $\tau_{i}>0$ that converges to zero, and a sequence of $\tau_{i}$-approximations $\mathfrak{p}_{i}: \mathcal{N}_{i} \rightarrow Y$ which induces a structure of locally trivial bundle on $\mathcal{N}_{i}$ whose fibers are either spheres or 2-dimensional tori.

\section{LEMMA 18}

The fibers of $\mathfrak{p}_{i}: \mathcal{N}_{i} \rightarrow Y$ are tori.

Proof

Since the fibers are either spheres or tori, it suffices to eliminate the first possibility. Hence, let us suppose for a contradiction that the fibers are spheres. Given $z_{1} \in Z-\mathcal{B}$ and a positive constant $\delta \ll \operatorname{diam}_{Z}(K)$, consider a sequence of points $q_{i} \in N_{i}-\Sigma$ converging to $z_{1}$ and such that $d_{H G}\left(q_{i}, z_{1}\right)<\delta$ for all $i \in \mathbb{N}$.

\section{CLAIM 19}

There exists $i_{0} \in N$ such that, for every $i>i_{0}$, we can find a homotopically nontrivial loop $\gamma_{i}$ (in $M-\Sigma$ ) based on $q_{i}$ whose length does not exceed $\delta$.

\section{Proof}

Consider the loops based on $q_{i}$ which are constituted by two minimizing geodesic segments with the same ends and having equal lengths bounded by $\frac{\delta}{2}$. Note that these loops are always homotopically nontrivial. To say that a point $q_{i}$ is not a base point for a loop as mentioned above is equivalent to saying that its injectivity radius is not less than $\frac{\delta}{2}$. This is a contradiction to the assumption of collapsing and therefore the claim follows. 
Now fix $i>i_{0}$, and consider a boundary sphere of $\mathcal{N}_{i}$ associated with an unbounded component of $Z-K$. By construction, this sphere separates $M$ into two components: one of these components contains the singular link $\Sigma_{0} \neq \emptyset$ (recall that $\Sigma_{1} \subset \Sigma_{0}$ by hypothesis) and the other contains a homotopically nontrivial loop as stated in the claim. Since $M-\Sigma$ is irreducible, this cannot happen, and therefore, the fibers must be solid tori. This establishes the lemma.

As in the 2-dimensional case, the components of $N_{i}-\mathcal{N}_{i}$ which have been associated with unbounded components of $Z-Y$ are tori. When $Z$ is homeomorphic to $[0, \infty)$, Shioya and Yamaguchi [SY, Theorem 0.5, Table 1] showed that the component of $N_{i}-\mathcal{N}_{i}$ associated with the bounded component of $Z-Y$ is homeomorphic to either a solid torus or a twisted $S^{1}$-bundle over a Möbius band Mö $\widetilde{\times} S^{1}$, that is, the quotient of $S^{1} \times[0,1] \times S^{1}$ by an involution

$$
\tau\left(e^{i \theta}, t, e^{i \eta}\right)=\left(e^{i(\theta+\pi)}, 1-t, e^{-i \eta}\right) .
$$

By being irreducible, $M$ is accordingly homeomorphic to a lens space or a prism manifold.

\subsubsection{Case where $Z$ has dimension 0}

This case was treated in [SY]. For the convenience of the reader, we state their result here.

\section{THEOREM 20 (COLLAPSING: DIMENSION 0)}

Suppose there is a point $p \in \Sigma_{1}$ such that the sequence $\left(M_{i}, p\right)$ converges to a 0 -dimensional pointed Alexandrov space $\left(Z, z_{0}\right)$. If $\sup \left\{R_{i}\left(\Sigma_{1}\right) ; i \in \mathbb{N}\right\}<\infty$, then $M$ is a Euclidean, spherical, or Nil manifold.

\subsection{Some consequences of Theorem 2}

Let $M$ be a closed, orientable, and irreducible 3-manifold, and let $\Sigma=\Sigma_{1} \sqcup \cdots \sqcup \Sigma_{l}$ be a fixed embedded link in $M$.

Given a convergent sequence $M_{i}$ of hyperbolic cone manifolds with topological type $(M, \Sigma)$, let

$$
\rho_{i}: \pi_{1}(M-\Sigma) \rightarrow \mathrm{PSL}_{2}(\mathbb{C})
$$

be an associated sequence of holonomy representations. Denote by $\operatorname{Vol}\left(\rho_{i}\right)$ the volume of the representation $\rho_{i}$ (see [Fra], [Dun] for definitions and details). It is well known that $\operatorname{Vol}\left(\rho_{i}\right)$ coincides with the Riemannian volume of $M_{i}-\Sigma$.

When the sequence $\operatorname{Vol}\left(\rho_{i}\right)$ converges to zero, the sequence $M_{i}$ necessarily collapses. It is a consequence of the definition. Unfortunately, it is not immediate that the lengths of singular links remain uniformly bounded. However, we have the following corollary. 


\section{COROLLARY 21}

If the sequence $\rho_{i}$ converges to a representation $\rho_{\infty}: \pi_{1}(M-\Sigma) \rightarrow \mathrm{PSL}_{2}(\mathbb{C})$ satisfying

$$
\operatorname{Vol}_{M-\Sigma}\left(\rho_{\infty}\right)=0
$$

(in particular, if $\rho_{\infty}$ is reducible), then $M$ is Seifert fibered or a Sol manifold.

Proof

According to the previous discussion, the sequence $M_{i}$ collapses. Since the convergence hypothesis trivially implies condition (1), the result is a direct consequence of Theorem 2.

Let us finish with the proof of Corollary 4. Due to Remark 3, the proof of Corollary 5 is the same.

\section{Proof of Corollary 4}

Let $M_{\alpha_{i}}$ be a convergent sequence as in the statement of the corollary. Since $M$ is a hyperbolic manifold, it is not Seifert fibered or a Sol manifold. Therefore, in the presence of hypothesis (2), it is a consequence of Theorem 2 that the sequence $M_{\alpha_{i}}$ cannot collapse.

\section{References}

[Bar] A. P. Barreto, Déformations de structures hyperboliques coniques, Ph.D. dissertation, Institut de Mathématiques de Toulouse, Toulouse, France, 2009 (accessed 8 October 2014), http://thesesups.ups-tlse.fr/838/1/Paiva-Barreto _Alexandre.pdf.

[Bel] I. Belegradek, Degenerations of Riemannian manifolds, preprint, arXiv:math/0701723v2 [math.DG].

[BLP] M. Boileau, B. Leeb, and J. Porti, Geometrization of 3-dimensional orbifolds, Ann. of Math. (2) 162 (2005), 195-290. MR 2178962.

DOI 10.4007/annals.2005.162.195.

[BBI] D. Burago, Y. Burago, and S. Ivanov, A Course in Metric Geometry, Grad. Stud. Math. 33, Amer. Math. Soc., Providence, 2001. MR 1835418. DOI $10.1090 / \mathrm{gsm} / 033$.

[BGP] Y. Burago, M. Gromov, and G. Perel'man, A. D. Aleksandrov spaces with curvatures bounded below, Russian Math. Surveys 47 (1992), 1-58. MR 1185284. DOI 10.1070/RM1992v047n02ABEH000877.

[Dun] N. M. Dunfield, Cyclic surgery, degrees of maps of character curves, and volume rigidity for hyperbolic manifolds, Invent. Math. 136 (1999), 623-657. MR 1695208. DOI 10.1007/s002220050321.

[Fra] S. Francaviglia, Hyperbolic volume of representations of fundamental groups of cusped 3-manifolds, Int. Math. Res. Not. IMRN 2004, no. 9, 425-459. MR 2040346. DOI 10.1155/S1073792804131619. 
[Fuj] M. Fujii, On strong convergence of hyperbolic 3-cone-manifolds whose singular sets have uniformly thick tubular neighborhoods, Kyoto J. Math. 41 (2001), 421-428. MR 1852992.

[Fuk] K. Fukaya, "Metric Riemannian geometry" in Handbook of Differential Geometry, Vol. II, Elsevier/North-Holland, Amsterdam, 2006, 189-313. MR 2194670. DOI 10.1016/S1874-5741(06)80007-5.

[Gro] M. Gromov, Structures métriques pour les variétés riemanniennes, Textes Math. 1, CEDIC, Paris, 1981. MR 0682063.

[HK2] C. D. Hodgson and S. P. Kerckhoff, Rigidity of hyperbolic cone-manifolds and hyperbolic Dehn surgery, J. Differential Geom. 48 (1998), 1-59. MR 1622600.

[HK] Universal bounds for hyperbolic Dehn surgery, Ann. of Math. (2) 162 (2005), 367-421. MR 2178964. DOI 10.4007/annals.2005.162.367.

[Kap] V. Kapovitch, "Perelman's stability theorem" in Surveys in Differential Geometry, Vol. XI, Surv. Differ. Geom. 11, Int. Press, Somerville, Mass., 2007, 103-136. MR 2408265. DOI 10.4310/SDG.2006.v11.n1.a5.

[Koj] S. Kojima, Deformations of hyperbolic 3-cone-manifolds, J. Differential Geom. 49 (1998), 469-516. MR 1669649.

[Sal] A. Salgueiro, Orders and actions of branched coverings of hyperbolic links, Topology Appl. 156 (2009), 1703-1710. MR 2521706.

DOI 10.1016/j.topol.2009.01.014.

[SY] T. Shioya and T. Yamaguchi, Collapsing three-manifolds under a lower curvature bound, J. Differential Geom. 56 (2000), 1-66. MR 1863020.

[Y2] _ "A convergence theorem in the geometry of Alexandrov spaces" in Actes de la Table Ronde de Géométrie Différentielle (Luminy, 1992), Sémin. Congr. 1, Soc. Math. France, Paris, 1996, 601-642. MR 1427772.

Universidade Federal de São Carlos, São Carlos, Brazil; alexandre@dm.ufscar.br 